\title{
Assessing an Improved Bayesian Model for Directional Motion Based Wave Inference
}

\author{
Jordi Mas-Soler ${ }^{1,2, *,+} \mathbb{D}$, Antonio Souto-Iglesias ${ }^{3,+} \mathbb{( D}$ and Alexandre N. Simos ${ }^{1,+}+\mathbb{D}$ \\ 1 Naval Arch. \& Ocean Eng. Department, Escola Politécnica, University of São Paulo (USP), \\ São Paulo-SP 05508-030, Brazil; alesimos@usp.br \\ 2 CEHINAV, ETSIN, Universidad Politécnica de Madrid (UPM), 28006 Madrid, Spain \\ 3 CEHINAV, DACSON, ETSIN, UPM, 28006 Madrid, Spain; antonio.souto@upm.es \\ * Correspondence: jordi@tpn.usp.br \\ + These authors contributed equally to this work.
}

Received: 15 February 2020; Accepted: 21 March 2020; Published: 26 March 2020

\begin{abstract}
An innovative Bayesian motion-based wave inference method is derived and assessed in this work. The evaluation of the accuracy of the proposed prior distribution has been carried out using the results obtained during a dedicated experimental campaign with a scale model an Oil and Gas (O\&G) semisubmersible platform. As for the Bayesian statistical inference approaches, the features of the proposed novel prior distribution, as well as the hypotheses adopted, are discussed. It has been found that significant improvements can be obtained if the new approach is adopted to estimate the sea conditions from measured vessel motions. Finally, it is possible to highlight a substantial reduction of the computing time when the sea conditions are estimated by means of the improved Bayesian method, if compared with the conventional approaches for motion-based wave inference.
\end{abstract}

Keywords: directional motion-based wave inference; semisubmersible platform; Bayesian modelling; prior distribution

\section{Motivation}

Traditionally, on-site measurements of the directional sea spectra were carried out by means of moored wave buoys. During the past decades, these measurement systems have been complemented by new technologies, such as wave radars and vessel motion-based wave inference systems (VMBs). More specifically, there has been a growing interest in the measurement of the directional sea spectrum, due to its inherent practical value for a wide range of offshore design and operational engineering activities.

Previous research works (see [1-3]) have shown that the three leading reasons why the VMBs have attracted much attention in the recent years are: (1) the good accuracy of the results obtained; (2) the low initial investment required ${ }^{1}$ and (3) the advantage that ships and offshore platforms can work together to provide worldwide information about the sea conditions, even from remote areas, at anytime.

Several works (e.g., [4-6]) have shown the accuracy of the estimations of the sea conditions obtained through the VMBs. However, in some sporadic cases, the authors also observed large discrepancies between the inferred directional sea spectra and the measurements adopted as the

1 VMBs are mainly composed by an ordinary set of accelerometers connected to a consumer-level PC, hardware that the major part of the oil and gas (O\&G) offshore platforms already have installed on-board 
paradigm for assessing the performance of the VMB. Ref [7] grouped these discrepancies as follows: (i) spurious energy peaks at the bow and stern of the vessel in beam sea conditions and (ii) global mean wave direction, when deviates from the expected value, has a consistent and systematic error.

In order to mitigate the misleading estimations of the directional sea spectra rendered by the VMBs, it would be desirable to properly address the uncertainties introduced by the Response Amplitude Operators (RAOs) and the regularization conditions adopted to solve the resulting seakeeping inverse problem. This topic will be addressed in this paper by means of an improved non-parametrical VMB model, which accuracy will be assessed using a scale model results of a semisubmersible O\&G platform.

\section{State of the Art}

Accurate estimations in preceding research work using non-parametrical VMBs (e.g., [1,8]) as well as the versatility of the technique to take into account several hypotheses have motivated the adoption of the Bayesian inference approach in this work to infer the directional sea spectra.

The core principle of the Bayesian inference is that the posterior distribution of the variable of interest (in this case the unknown directional sea spectrum) is the result of updating the existing prior information on the model parameters using the available data (measured motions of the floating vessel), where the systematic process of learning from the data is implemented via Bayes theorem. Since there exists a significant body of research regarding the Bayesian VMBs, the full mathematical treatment will not be reviewed in detail in this paper. For the reader who is interested in a comprehensive description of this inference approach, it is important to mention that a concise derivation of the formulae can be found in, among others, Refs. [8,9].

Model scale experiments have been crucial for the development of the Bayesian VMBs and to validate the prior information adopted concerning the sea spectra. In particular, Refs. $[4,10]$ have been significant works for this area. The experiments have referred to moored O\&G structures, which implied that the research conducted had to focus on developing a proper Bayesian inference approach to estimate the sea conditions. The estimations were performed using the Bayesian inference method with three prior parameters, analogous to the method proposed by Nielsen [1]. Simos [10], however, proposed a relevant conceptual change. Different from the original proposal, where the hyperparameters of the prior distribution were estimated by means of the ABIC method (see [1]), the authors set a pre-calibration of the prior hyperparameters, meaning that their values were prescribed for each wave estimation.

Regarding full-scale experimental campaigns, Simos [2] and daSilva [7] have conducted systematic experimental validations using data measured from FPSOs operating in Brazil's Campos and in Peregrino field. During these validations campaigns, the outputs of the inference approach were compared with the hindcast measurements provided by NOAA for the same region and a commercial marine radar system, showing that the Bayesian inference approach with a set of pre-determined prior hyperparameters is able to accurately estimate the sea conditions.

At the same time, daSilva [7] highlighted that there are some difficulties associated with the measuring of the wave conditions through the VMB. Indeed, different drawbacks may arise for certain sea conditions (usually extreme weather conditions when non-linear responses of the vessel become relevant). The literature addressing this shortcoming is limited. Iseki [11] aims at introducing the RAOs estimation inaccuracies in a Bayesian VMB by means of a RAO uncertainty matrix, which was adopted ad hoc to obtain accurate solutions from full-scale data. Mas-Soler [9] followed a similar approach, in which the RAOs uncertainty matrix was determined by means of the coherence functions from model scale data. However, these approaches do not deal with some inherent difficulties associated with the measuring of the wave conditions through the VMB as adopted by Simos [2], such as the time-consuming process of the prior hyperparameters pre-calibration and the adoption of three hyperparameters. 
Finally, the estimations obtained by Mas-Soler [3], using the same semisubmersible platform model and the Bayesian VMB as provided in Ref. [2], will be used in this work as a reference for the assessment of the reliability of the proposed approach.

Considering this motivation and review of previous research, the following topics will be investigated in the paper:

1. How can the prior distribution be modified in order to take into account the shortcomings highlighted in the literature?

2. How can an alternative prior distribution provide practical improvements for the Bayesian VMBs?

The proposed approach to meet each one of these topics includes:

1. Assessment of the main limitations of the Bayesian models adopted in previous works for VMBs, followed by the derivation of an alternative prior distribution.

2. Compare results inferred with the proposed alternative prior distribution with the results obtained by means of the conventional Bayesian VMB, as proposed in Ref. [2].

The paper is organized as follows: first, the outline of the theoretical approach. This section is followed by the experimental setup and test matrices proposed. The fifth section includes the main results and their discussion. Finally, the last section is a summary of the main conclusions reached through this work, which is complemented with some recommendations for future work.

\section{Outline of the Theoretical Approach}

The qualitative introduction to the Bayesian inference provided in section 2 can be formally expressed as:

$$
P(x \mid B)=\frac{P(B \mid x) p(x)}{P(B)} \propto P(B \mid x) p(x),
$$

where $P(x \mid B)$ is the posterior distribution, $P(B \mid x)$ the probability of observing $B$ given $x, p(x)$ is the prior distribution and $P(B)$ stands for the marginal probability distribution of observing the event $B$.

The adoption of the Bayesian inference approach in VBMs is based on the assumption that the relation between the wave excitation forces and the vessel responses is linear and, therefore, the cross spectra of the motion time series can be formally expressed through the following identity:

$$
\phi_{i j}(\omega)=\int_{-\pi}^{\pi} Z_{i}(\omega, \beta) Z_{j}^{*}(\omega, \beta) S(\omega, \beta) \mathrm{d} \beta,
$$

where $Z_{i}(\omega, \beta)$ is the RAO of the $i^{t h}$ motion at wave frequency $\omega$ and direction of incidence $\beta . Z_{j}^{*}(\omega, \beta)$ stands for the complex conjugate of the RAO of the $j^{t h}$ motion and $S(\omega, \beta)$ is the directional wave spectrum. This identity can be expressed in discrete form assuming the integrand to be constant $\left(\Delta \beta=\frac{2 \pi}{K}\right)$ :

$$
\phi_{i j}(\omega)=\Delta \beta \sum_{k=1}^{K} \mathrm{Z}_{i}\left(\omega, \beta_{k}\right) \mathrm{Z}_{j}^{*}\left(\omega, \beta_{k}\right) S\left(\omega, \beta_{k}\right),
$$

where $\beta_{k}=-\pi+(k-1) \Delta \beta$ and $K$ is the number of discrete wave directions considered. Concerning the indices $i$ and $j$, they correspond to the dofs (degrees of freedom). For the sake of illustration, in this work these indices vary between 3 and 5 , since only three motions of the floating vessel are adopted $(N=3)$, namely: heave, roll and pitch.

If a certain range of $M$ wave frequencies is specified previously, being $\Delta \omega=\frac{\omega_{M}-\omega_{1}}{M-1}$, Equation (3) can be expressed in a matrix form as follows:

$$
\mathbf{B}=\mathbf{A} \mathbf{x}+\varepsilon .
$$

where the vector $\mathbf{B}$ contains a total of $\left(N^{2} \cdot M \times 1\right)$ elements, which are related to the measured motion spectra. The RAO matrix is represented by the matrix $\mathbf{A}$, which is formed by a diagonal of $M$ 
matrices, each one with $\left(N^{2} \cdot M\right) \times(K \cdot M)$ elements, vector $\mathbf{x}$ stands as the unknown sea spectrum, evaluated at the $(K \times M)$ pairs of frequencies and directions and $\varepsilon$ is a vector with $\left(M \cdot N^{2} \times 1\right)$ elements representing the measurement error, which has been assumed to be Normally distributed, with zero mean value and variance $\sigma^{2}$. For a comprehensive description of the quantities $\mathbf{B}, \mathbf{A}$ and $\mathbf{x}$ the reader is referred to [1].

The estimation of the vector $\mathbf{x}$ is obtained maximizing the product of the likelihood function $L(\mathbf{x} \mid B)$ by the prior distribution, where the likelihood function represents the probability of occurrence of the spectrum given a specific measurement $B$.

$$
L(\mathbf{x} \mid B)=\left(\frac{1}{2 \pi \sigma^{2}}\right)^{\frac{N^{2}}{2}} \exp \left(-\frac{1}{2 \sigma^{2}}\|B-A \mathbf{x}\|^{2}\right) .
$$

Following the idea presented in [2], the posterior distribution for Bayesian VMBs is hence written as:

$$
L(\mathbf{x} \mid \mathbf{B}) \cdot p(\mathbf{x})=\left(\frac{1}{2 \pi \sigma^{2}}\right)^{\frac{N^{2}}{2}} \exp \left(-\frac{1}{2 \sigma^{2}}\left[\|\mathbf{B}-\mathbf{A x}\|^{2}+\mathbf{x}^{T}\left(u_{1}^{2} \mathbf{H}_{1}+u_{2}^{2} \mathbf{H}_{2}+u_{3}^{2} \mathbf{H}_{3}\right) \mathbf{x}\right]\right) .
$$

The matrices $\mathbf{H}_{1}$ and $\mathbf{H}_{2}$ stand as the operators, in direction and frequency domains, that introduce the regularization condition (i.e., the estimated sea spectrum must be smooth and continuous) by means of second partial differences between neighbor values. The matrix $\mathbf{H}_{3}$ is formally similar to the previous ones, but in this case, it is only applied in the high-frequency range where the vessel is expected to present small (almost negligible) response amplitudes. Concerning $u_{1}, u_{2}$ and $u_{3}$, they stand for the pre-determined prior hyperparameters (see, once again, Ref. [2]).

\subsection{Alternative Prior Distribution}

This section contains a detailed explanation regarding the proposal of an alternative prior distribution for the Bayesian inference method. Nonetheless, a comprehensive description of the main shortcomings of the approach adopted in Refs. [2,7] is provided in order to enable the reader to understand the main motivations that led to this proposal.

Limitations of the conventional approach

The results provided by daSilva [7] highlighted the good accuracy of the Bayesian method to estimate the sea conditions that happened during the experimental, provided they were in the estimation range (i.e., as the authors noted, $T_{p}>8 \mathrm{~s}$ ). However, occasional discrepancies between the estimations obtained and the radar measurements (i.e., paradigm) have been identified.

It is plausible to consider that part of the misleading estimations of the sea conditions can be explained by limitations of the prior distribution adopted by Simos [2]. Possible improvements of the prior distribution are:

- It is known from the formal discussion provided in Ref. [12], that the prior distribution should be determined taking into account the measured data. Although previous works have been based on a pre-calibration procedure of the hyperparameters, the current work aims at providing a prior distribution which is computed using the measured data without compromising the practical features gained with the pre-calibration of the hyperparameters;

- The likelihood function adopted works as a high-pass filter and it has the undesirable effect of amplifying the measurement uncertainties when the ship is expected to present no significant motions. Thus, a mechanism capable of properly computing the variance introduced by the points of minima of the RAOs would be welcome;

- The pre-definition of the two first hyperparameters, $\left(u_{1}, u_{2}\right)$, showed several advantages, e.g., the algorithm is less time-consuming and a consistent improvement of the estimation has been 
observed. However, in some cases, the values adopted for the hyperparameters are not optimal (see [9]). This effect may be related to the point of minima of the RAOs, where the vessel is expected to have no significant responses, but certain wave conditions may impose some motions leading to poor estimations of the sea state. In addition, this approach requires a pre-calibration procedure that involves numerical simulations and, in some cases, real scale data to validate the values estimated with the hyperparameters chosen. The novel prior distribution proposed does not rely on the use of these hyperparameters as proposed in previous works and, therefore, it is expected that this characteristic of the prior distribution will help to avoid some of the drawbacks related to the pre-defined hyperparameters;

- Despite the fact that the use of the third hyperparameter, $\left(u_{3}\right)$, provides a useful technique to avoid spurious energy estimations, this methodology restrains the estimation of the energy in certain frequency ranges in which the ship still has representative motions and this can have a negative impact on the estimations of the significant wave height and mean period. The prior distribution proposed avoids the use of a third hyperparameter, whose influence on the final solution has been modeled by means of a method that avoids misleading energy estimations within the frequency limits.

From the points listed above, the following can be concluded: For mitigating the errors of estimation, the uncertainties introduced by the RAOs should be properly addressed by the prior distribution. A method to calibrate the trade-off between the likelihood function and the prior distribution is needed, at least one that would lead to optimal estimations of the prior distribution in the majority of the cases.

An heuristic prior distribution

Let us assume that the prior distribution function of the unknown spectrum, $S_{\eta \eta}$, is given by a negative exponential probability distribution in the form of:

$$
p(\mathbf{x}) \propto \exp \left\{-\frac{\|\mathbf{C} \mathbf{x}\|^{2}}{2 \sigma^{2}}\right\},
$$

where $\mathbf{C}$ stands as a matrix of coefficients that must be estimated. In order to help the reader to better understand the alternative approach described in this section, Figure 1 provides a general overview of the procedure followed to derived matrix $\mathbf{C}$.

Returning to the prior distribution, the previous assumption allows us to estimate the variance of the $j^{\text {th }}$ element (with $j$ ranging between 1 and $K \cdot M$ ) of the unknown sea spectrum, $S_{\eta \eta_{j}}$, as follows (see [13]):

$$
\operatorname{Var}\left(S_{\eta \eta_{j}}\right)=\mathbf{e}_{j}\left(\int_{\mathbb{R}^{M+1}} S_{\eta \eta}^{2} p\left(S_{\eta \eta}\right) \mathrm{d} S_{\eta \eta}\right) \mathbf{e}_{j}^{T}=\sigma^{2} \mathbf{e}_{j}\left(\mathbf{C}^{T} \mathbf{C}\right)^{-1} \mathbf{e}_{j},
$$

where $\boldsymbol{e}_{j}$ stands for the $j^{\text {th }}$ canonical basis vector in $\mathbb{R}^{M+1}$.

Accordingly,

$$
C_{j j}=\left(\frac{\sigma^{2}}{\operatorname{Var}\left(S_{\eta \eta_{j}}\right)}\right)^{\frac{1}{2}} \text { and } C_{i j}:=0,
$$

Let us assume that an initial estimate of the sea spectrum $(\hat{\mathbf{x}})$ is somehow known. It is possible to state that the variance of the largest elements of the sea spectrum is larger than the variance of the smallest ones. Accordingly, it has been assumed, heuristically, that the variance of each element of the unknown spectrum is proportional to,

$$
\operatorname{Var}\left(S_{\eta \eta_{j}}\right) \propto \frac{\max (\hat{\mathbf{x}})-\min (\hat{\mathbf{x}})}{\left|\hat{\mathbf{x}}_{j}-\max (\hat{\mathbf{x}})\right|}
$$


Now, because the regularization term can be assimilated to an approximation of the measurement error and aiming to facilitate the computing process, the final expression of each element of matrix $\mathbf{C}$ has been adopted as:

$$
C_{j j} \cdot C_{j j}=\frac{\left|\hat{\mathbf{x}}_{j}-\max (\hat{\mathbf{x}})\right|}{\max (\hat{\mathbf{x}})-\min (\hat{\mathbf{x}})} \sigma^{2} .
$$

Therefore, the computation of the matrix $\mathbf{C}$ is reduced to obtain an initial estimate of the sea spectrum $\hat{x}$, that is also needed to estimate the variance of the measurement error, $\sigma^{2}$, as it is shown below. This results in a regularization term that induces larger estimation errors in the frequency regions in which the sea spectrum presents small amounts of power or the responses of the platform are not significant, i.e., the uncertainty of the linear model is larger when the platform does not present significant motions.

In this case, the prior distribution relies on the computation of an initial estimation of the sea spectrum, which will be estimated from the measured data. It is known that the use of the measured data to construct the prior distribution can introduce different correlations between the likelihood function and the prior distribution, resulting in misleading estimations of the sea conditions. Aiming to avoid any bias on the posterior distribution, as well as to maintain the benchmark of the prior distribution proposed in previous works, the ABIC method has been adopted.

Introducing the posterior distribution, as proposed by Nielsen [1], in the general form of the ABIC [12]. The following relation for the ABIC can be obtained:

$$
A B I C=N(N+1) \log \left(2 \pi \sigma^{2}\right)-K \cdot M \lg \left(\lambda^{2}\right)-2 \log \int\left(\frac{1}{2 \pi \sigma^{2}}\right)^{\frac{K M}{2}} \exp \left\{\frac{-1}{2 \sigma^{2}} J(\hat{\mathbf{x}})\right\},
$$

which can be rearranged as:

$$
A B I C=N(N+1) \log \left(2 \pi \sigma^{2}\right)-K \cdot M \lg \left(\lambda^{2}\right)+\frac{J(\hat{\mathbf{x}})}{\sigma^{2}}+\mathcal{F}(\lambda, \mathbf{A}, \mathbf{D}),
$$

where $N, M$ and $K$ are constants related to the number of variables and $J(\hat{\mathbf{x}})=\|\mathbf{B}-\mathbf{A} \hat{\mathbf{x}}\|^{2}+\hat{\mathbf{x}}^{T}\left(u_{1}^{2} \mathbf{H}_{1}+\right.$ $\left.u_{2}^{2} \mathbf{H}_{2}\right) \hat{\mathbf{x}}$.

In order to compute the value of $\sigma^{2}$ that minimizes the ABIC, Equation (13) is differentiated with respect to the variable of interest $\left(\sigma^{2}\right)$ and then set equal to zero, leading to:

$$
\sigma^{2}=\frac{J(\hat{\mathbf{x}})}{N(N+1)}=\frac{\|\mathbf{B}-\mathbf{A} \hat{\mathbf{x}}\|^{2}+\hat{\mathbf{x}}^{T}\left(u_{1}^{2} \mathbf{H}_{1}+u_{2}^{2} \mathbf{H}_{2}\right) \hat{\mathbf{x}}}{N(N+1)} .
$$

Since the matrix $\mathbf{C}$ is expected to provide an estimation of the variance of measurement error, the values of the hyperparameters of Equation (14) were set equal to 1, that also is consistent with hypothesis that led to Equation (11). Finally, an estimation of $J(\hat{\mathbf{x}})$ is needed in order to compute the variance of the measurement error. In this case, it has been computed as:

$$
\hat{\mathbf{x}}=\left(\mathbf{A}^{T} \mathbf{A}+1^{2} \mathbf{H}_{1}+1^{2} \mathbf{H}_{2}\right)^{-1} A^{T} \mathbf{B} .
$$




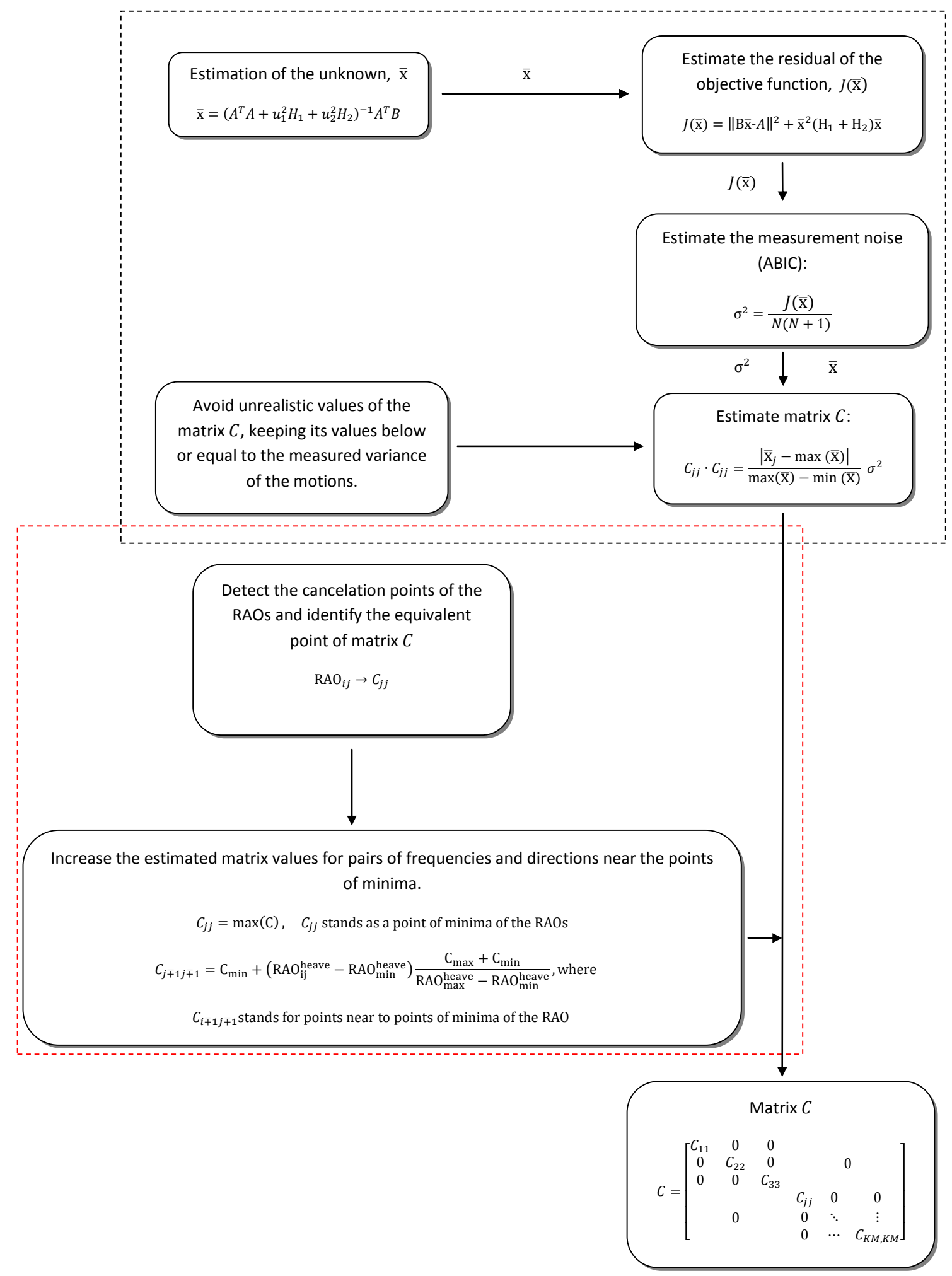

Figure 1. Schematic representation of the algorithm adopted to estimate the matrix $\mathbf{C}$. 
Since we are trying to approximate the variance of the measurement error and Equation (15) provides the minimum of the objective function under the hypotheses adopted, from Ref. $[14]^{2}$ we obtain that a single estimation of the residual of the objective function is enough, making the process less time-consuming. It is worthy to highlight that the objective function selected will be indirectly included in the prior distribution and, as a consequence, the adoption of this objective function assures a certain preference to smooth solutions. Nonetheless, at the same time, it acts as a weak condition in contrast with the maximization problem proposed by Nielsen [15].

The resulting procedure may provide large values of $\mathbf{C}$. In order to avoid unbounded values that would result in an unstable prior distribution, the values of the elements of matrix $\mathbf{C}$ will be kept equal to or below a certain threshold, as shown next. In this particular case, it is possible to state that the variance of the measurement error is always equal or smaller than the variance of the measured motion that is expected to provide the largest response amplitudes (i.e., heave) and referred as, $\sigma_{B}$. Since the value of the estimated $\mathbf{C}$ is expected to vary between 0 and $\sigma^{2}$ it is possible to write the following relation,

$$
C_{j j} \cdot C_{j j}=\frac{\left|\hat{\mathbf{x}}_{j}-\max (\hat{\mathbf{x}})\right|}{\max (\hat{\mathbf{x}})-\min (\hat{\mathbf{x}})} \sigma^{2} \leq \sigma_{B}^{2} .
$$

An estimation of $\mathbf{C}$ can be obtained by substituting the output of Equations (14) and (15) into Equation (16). However, this procedure, once again, may lead to unbounded values for the estimation of the unknown for the pairs of frequencies and directions linked to the point of minima of the RAOs. As mentioned above in this section, waves with frequencies and directions that correspond to cancellations points of the RAOs, when the vessel is expected to have no significant response, may, actually, impose some motions on the platform and result in the increase of the method's inaccuracy. This is because the numerical model for the RAOs is not free from errors. The procedure described below avoids this shortcoming,

1. Components $C_{j j}$ of matrix $C$ linked to the cancellation points of the RAOs are set equal to the maximum of the output obtained by means of the estimation of matrix $\mathbf{C}$.

2. Components of matrix $\mathbf{C}$ near to the cancellation points and points of minima of the RAOs may provide, in certain conditions, unrealistic estimations of the wave spectrum. Therefore, if the corresponding values of $\mathbf{C}$ are less than a certain threshold, then the values are replaced by this threshold. The estimation of this threshold is done be means of a linear interpolation between the maximum and minimum of the matrix $\mathrm{C}$ accordingly to the heave $\mathrm{RAO}$, which has been select among the (six) RAOs due to the fact that it can be considered as an almost ideal response model, thus providing a better prediction of the responses of the platform in comparison with the other dofs.

With regard to the numerical considerations, the resulting matrix $\mathbf{C}$ will have the characteristics described in the following.

$$
C=\left[\begin{array}{cccccc}
C_{11} & 0 & 0 & \cdots & 0 & 0 \\
0 & C_{22} & 0 & \cdots & 0 & 0 \\
0 & 0 & \ddots & \cdots & 0 & 0 \\
\vdots & \vdots & \vdots & C_{j j} & 0 & 0 \\
0 & 0 & 0 & 0 & \ddots & \vdots \\
0 & 0 & 0 & 0 & \cdots & C_{K M, K M}
\end{array}\right] \text {, with } \quad C_{j j} \cdot C_{j j}=\frac{\left|\hat{\mathbf{x}}_{j}-\max (\hat{\mathbf{x}})\right|}{\max (\hat{\mathbf{x}})-\min (\hat{\mathbf{x}})} \sigma^{2} \leq \sigma_{B}^{2}
$$

2 That work proved consistency and asymptotic normality of the maximization objective function under relatively weak conditions concerning the exact distribution of the likelihood function and the use of the second derivatives of the likelihood function as the regularity conditions. 
The alternative prior distribution results in the following posterior distribution:

$$
L(\mathbf{x} \mid \mathbf{B}) \cdot p(\mathbf{x})=K \cdot \exp \left(-\frac{1}{2 \sigma^{2}}\left[\|\mathbf{B}-A \mathbf{x}\|^{2}+\|C \mathbf{x}\|^{2}\right]\right) .
$$

As it has been pointed out before, the total size of the maximization problem is still defined by the number of directions $(K)$ and frequencies $(M)$ considered, being the number of variables equal to $K \times M$. Therefore, the solution of the problem is given by the minimization of the following objective function:

$$
J(\mathbf{x})=\|\mathbf{B}-A \mathbf{x}\|^{2}+\|C \mathbf{x}\|^{2} .
$$

This minimization is feasible by means of a conventional quadratic programming algorithm.

\section{Description of the Experimental Campaign}

A 1:120 scale model of an O\&G semisubmersible platform was used during the dedicated experimental campaign carried out at the wave basin of the Universidade de São Paulo (TPN-USP). The structure of the model is mainly composed of a square ring pontoon connected to the deck by means of six box-shaped columns. A comprehensive description of the main characteristics of the platform assessed in this work are provided in Ref. [16] and summarized in Table 1. In order to guarantee that the desired mass-inertia (project) features of the platform model were achieved, a series of pre-tests were carried out during the experimental campaign. This series of pre-test included bifilar pendulum tests, static inclination tests, decay tests and regular wave tests.

The tests included 32 different irregular long-crested sea conditions, ranging from mild waves with high probability of occurrence to extreme $100 \mathrm{yr}$-return events, with a full-scale equivalent time duration of 1.5 hour, each. Following the guidelines provided by Torsethaugen [17], for each pair of $H_{s}$ and $T_{p}$ the wave generated at the wave basin followed the most convenient sea spectrum, namely Torsethaugen or JONSWAP. For a comprehensive description of the experimental campaign and the criterion followed to select the most convenient sea spectrum for each sea condition tested at the TPN-USP the reader is referred to Ref. [3]. Aiming at providing a general idea of the waves generated during the experimental campaign, Figure 2 shows the model-scale of the semisubmersible platform during an irregular wave test.

Table 1. Geometric properties of the semisubmersible platform.

\begin{tabular}{lcc}
\hline Properties & Full-Scale & Small-Scale \\
\hline LOA $(\mathrm{m})$ & 102.4 & 0.853 \\
$\mathrm{~B}(\mathrm{~m})$ & 96.0 & 0.800 \\
$\mathrm{D}(\mathrm{m})$ & 45.0 & 0.375 \\
Draft $(\mathrm{m})$ & 25.0 & 0.208 \\
Displacement $(\mathrm{kg})$ & $8.27 \cdot 10^{7}$ & 46.621 \\
Heave natural period (s) & 24 & 2.19 \\
Pitch natural period (s) & 88 & 8.03 \\
Roll natural period (s) & 74 & 6.75 \\
\hline
\end{tabular}




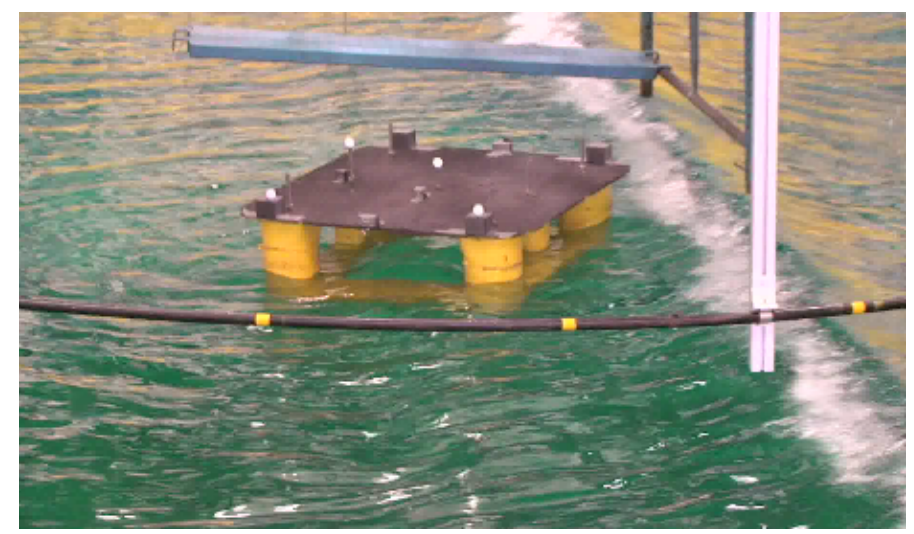

Figure 2. Picture of the model-scale semisubmersible platform during the tests carried out at the wave basin at Universidade de São Paulo (TPN-USP).

The results discussed in this work comprise only one draft (operational) and five-wave headings $\left(180^{\circ}, 150^{\circ}, 135^{\circ}, 120^{\circ}\right.$ and $\left.90^{\circ}\right)$. Since one end of the tank acted as an actuator (generating the waves) and the other end was used as an active wave-absorbing surface for all the sea conditions tested. Finally, it is important to remember that during the tests, the motions of the model were recorded with an optical tracking system, that stands as a non-intrusive measurement system with linear displacement uncertainty equal to $0.1 \mathrm{~mm}$.

The dynamic model adopted to infer the sea conditions is provided by the RAOs of the heave, roll and pitch motions, which were computed by means of the software WAMIT [18]. Concerning the dynamics of the semisubmersible platform, they are characterized by small damping coefficients, resulting in that the expected responses of the platform close the motion resonances are mainly driven by non-linear damping effects. In order to avoid an excessive loss of accuracy using the Bayesian $\mathrm{VMB}$, a simple estimation of the external damping for the heave, motion was obtained following the proposal derived in Ref. [3].

Figure 3 illustrates the good level of agreement obtained between the experimental motion RAOs and those computed numerically. The results provided comprise only the three motions that were used for the purpose of wave inference (namely: heave, roll, pitch). Regular and transient wave tests were used to check the accuracy of the motion RAOs obtained with the numerical model. Concerning the regular waves, due to the technical limitations of the wave basin, the estimations of the RAOs are limited to $4 \%$ and $6 \%$ steepnesses for a period equal to $22 \mathrm{~s}$. Regarding the heave motion RAOs, they were computed with damping ratios: $\zeta=5 \%, \zeta=3.81 \%$ and $\zeta=6.89 \%$. The damping ratios equal to $3.81 \%$ and $6.89 \%$ were estimated following the procedure derived in Ref. [3]. Since the transient wave tests were used to verify the accuracy of the numerical RAOs, the wave steepness was kept below $4 \%$ during all the tests. The range of periods for the generation of the transient waves was set from 8 to $24 \mathrm{~s}$ (in real scale), with a constant wave amplitude equal to $1.68 \mathrm{~m}$. Finally, the results provided show that significant differences in the vertical motions of the platform may appear due to viscous effects for high wave periods. These effects include, along with the attenuation of the resonant amplification, the suppression of the cancellation point of the heave's RAO. The latter effect results from the velocity-dependent drag forces. Thus, it cannot be reproduced by merely adding an external damping. 

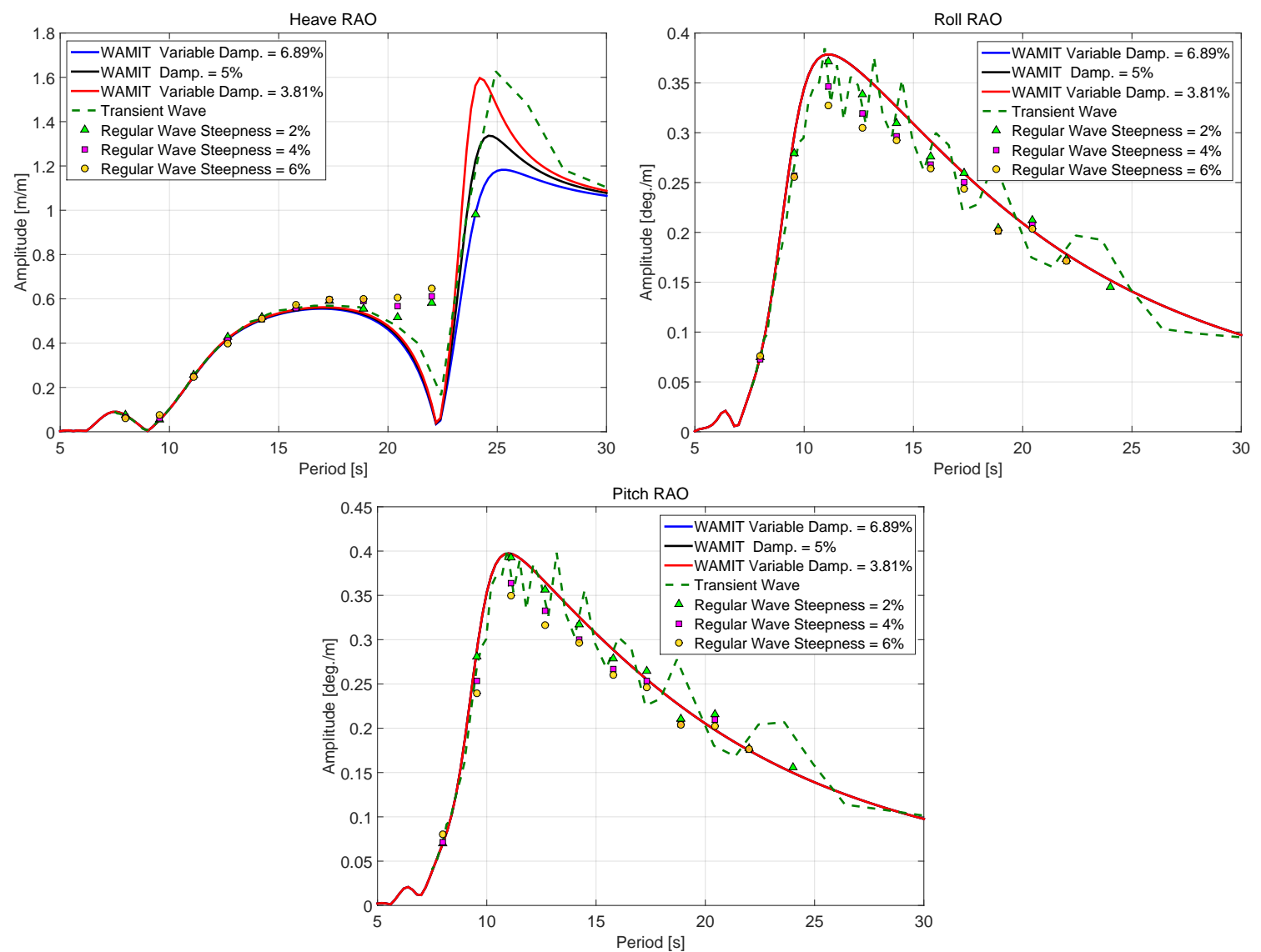

Figure 3. Heave, roll and pitch Response Amplitude OperatorsRAOs estimated numerically (black and blue lines), experimental RAOs obtained using transient (green dashed line) and regular waves with $2 \%, 4 \%$ and $6 \%$ steepnesses (colored markers).

\section{Results}

This section includes the main results obtained by means of the alternative prior distribution derived in this work. Aiming at assessing the accuracy of the estimations obtained, the outputs of the proposed non-parametrical VMB have been compared with the results obtained in [3], where the authors adopted the VMB approach as proposed in [2] and it is referred from now on as the conventional approach.

First, the accuracy of the estimations has been checked considering the main statistical parameters of the sea states, i.e., the mean wave period $\left(T_{1}\right)$, significant wave height $\left(H_{s}\right)$ and mean wave direction $(\theta)$. In order to provide an effective and proper visual formatting for the comparison of the results, the following arrangement is adopted: A representation of the distribution of the ratio between the value of the estimation and the measured value, for all the heading conditions $(90 ; 120 ; 135 ; 150$ and $180 \mathrm{deg}$ ), in terms of box-plots. Moreover, the results have been clustered into two sets with different colors, indicating whether the waves generated in the basin corresponded to a JONSWAP (blue) or Torsethaugen (red) power spectrum. The sea wave statistics $H_{s m}, T_{1 m}$ and $\theta_{m}$ stand for the significant wave height, mean wave period and mean wave direction obtained from the measurements in the basin using a set of conventional wave probes.

Figures $4-6$ show the results obtained by means of the conventional prior distribution. It is interesting to point out that there is an improvement of the estimations for sea conditions featured with large mean wave periods, tendency previously highlighted in [3], This is a trend that can be explained by the fact that in extreme sea conditions a large amount of information (the platform is catheterized with larger motion amplitudes) is provided by the platform motions. 


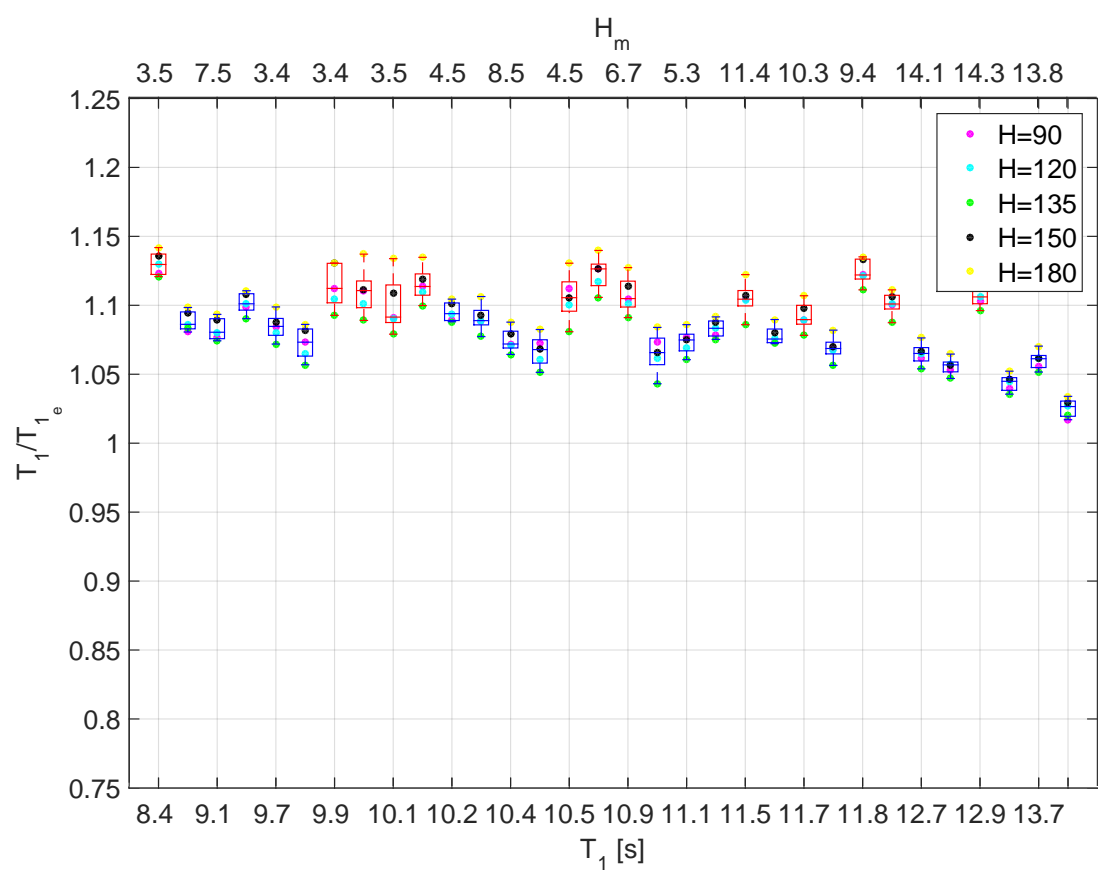

Figure 4. Box-plot showing the ratio between the estimated and measured mean wave period, obtained by means of the conventional approach.

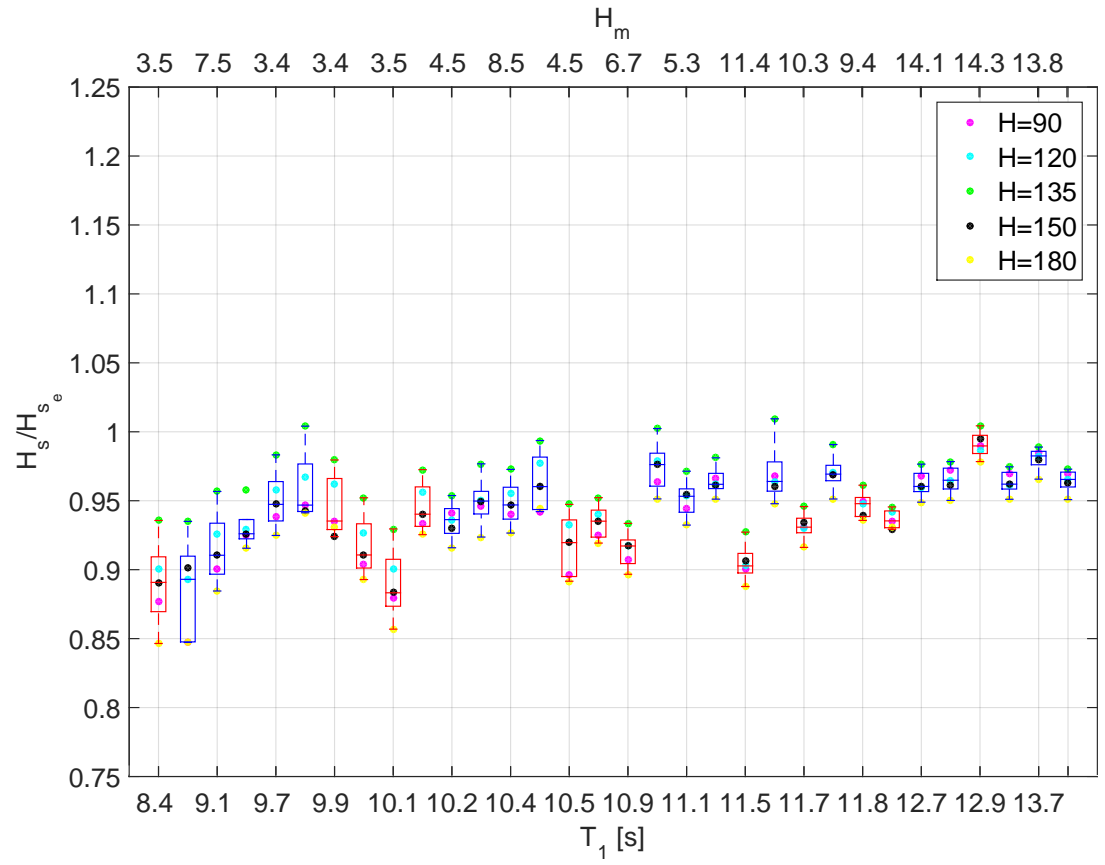

Figure 5. Box-plot showing the ratio between the estimated and measured significant wave height, obtained by means of the conventional approach. 


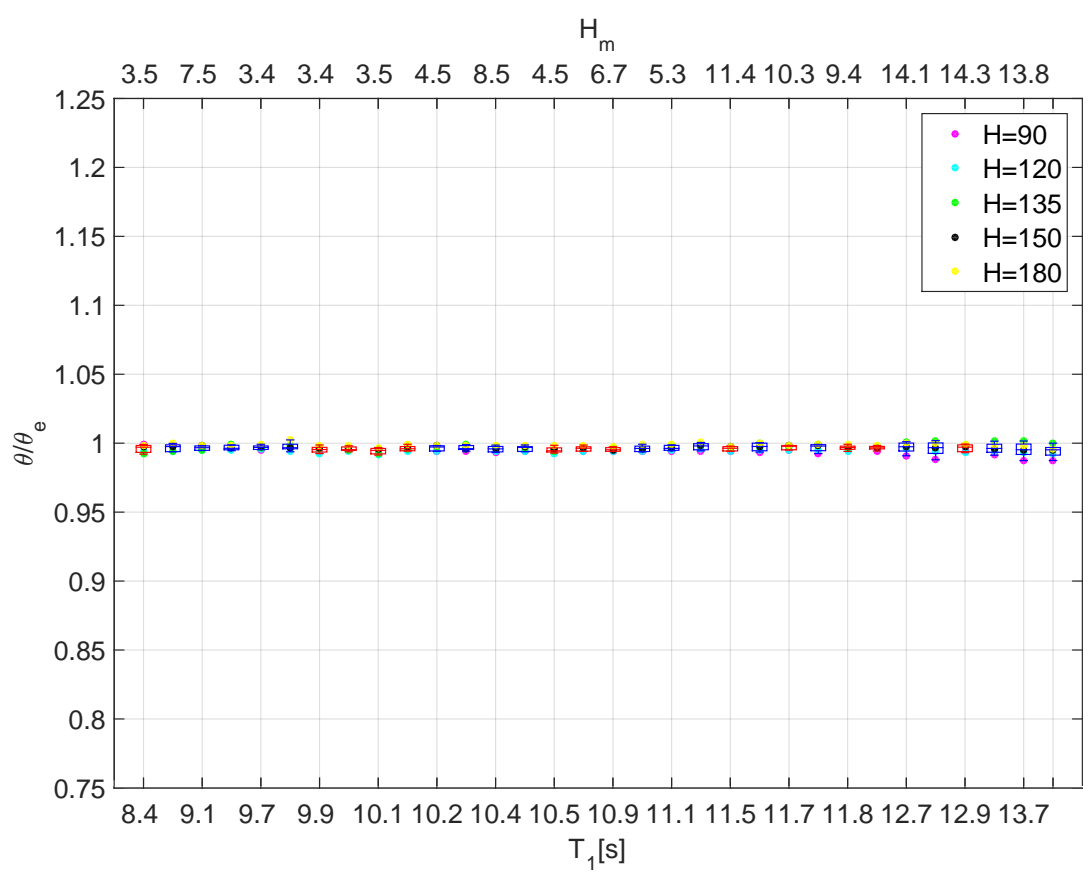

Figure 6. Box-plot showing the ratio between the estimated and measured mean wave direction, obtained by means of the conventional approach.

Similarly, Figures 7-9 provide the results obtained using the alternative prior distribution. In general, the results obtained show a clear improvement of the estimations regarding the mean wave period (see Figures 4 and 7 , respectively), $T_{1}$. Differently from the tendency identified in previous works (see [9]), the alternative approach seems to slightly underestimate the mean wave period. This was expected since the alternative prior distribution allows the estimations of larger amounts of energy in the high-frequency range.

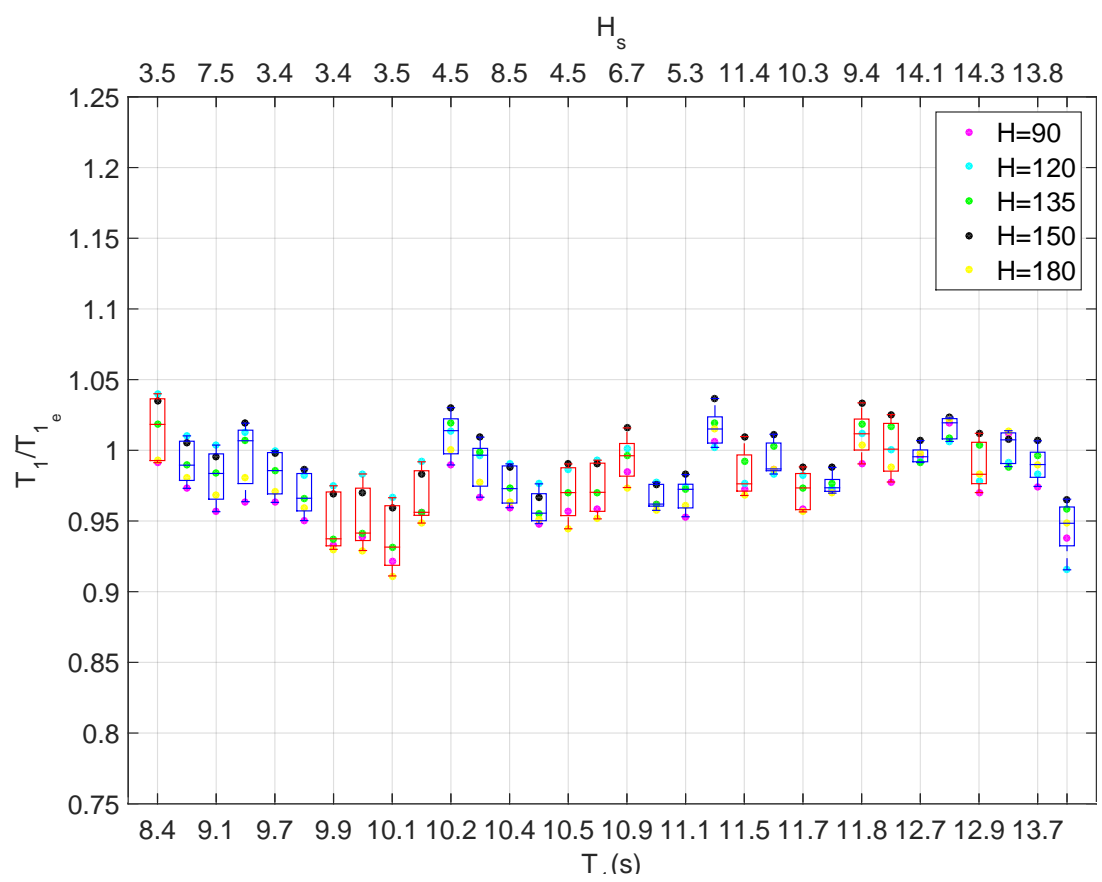

Figure 7. Box-plot showing the ratio between the estimated and measured mean wave period, obtained by means of the alternative prior distribution. 


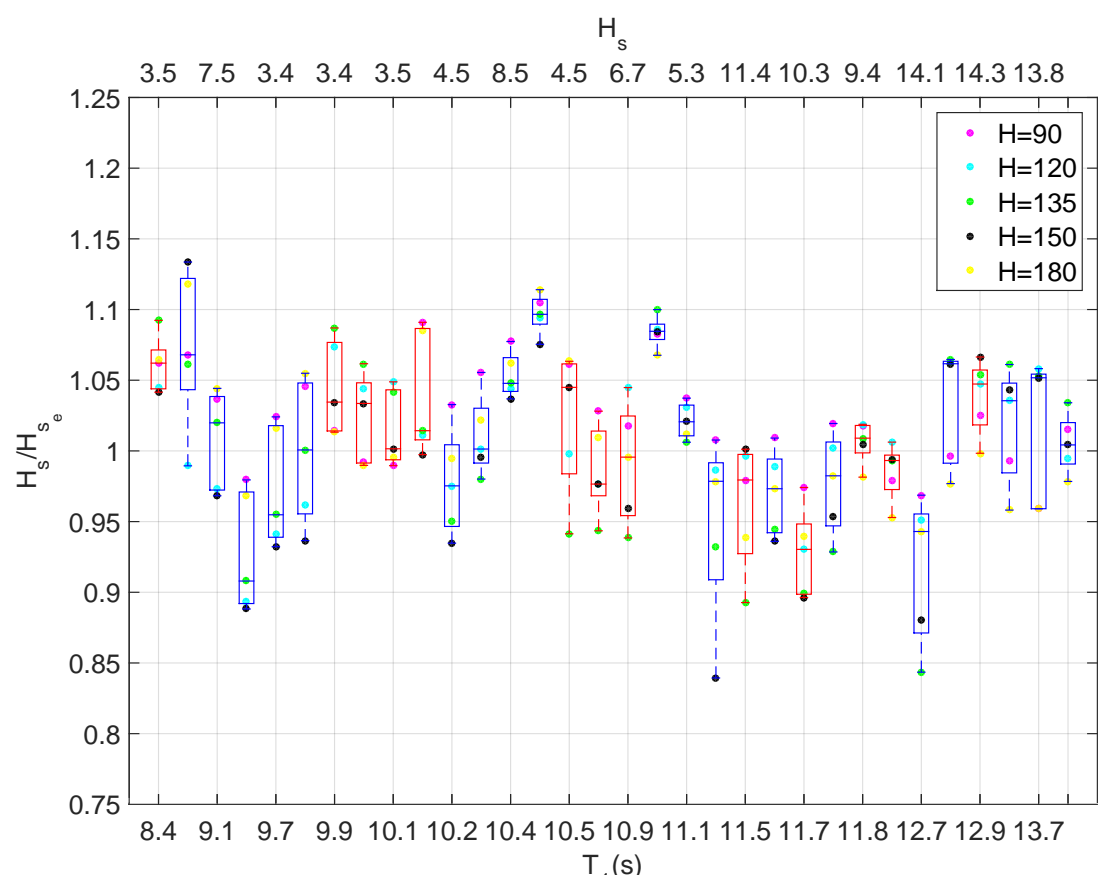

Figure 8. Box-plot showing the ratio between the estimated and measured significant wave height, obtained by means of the alternative prior distribution.

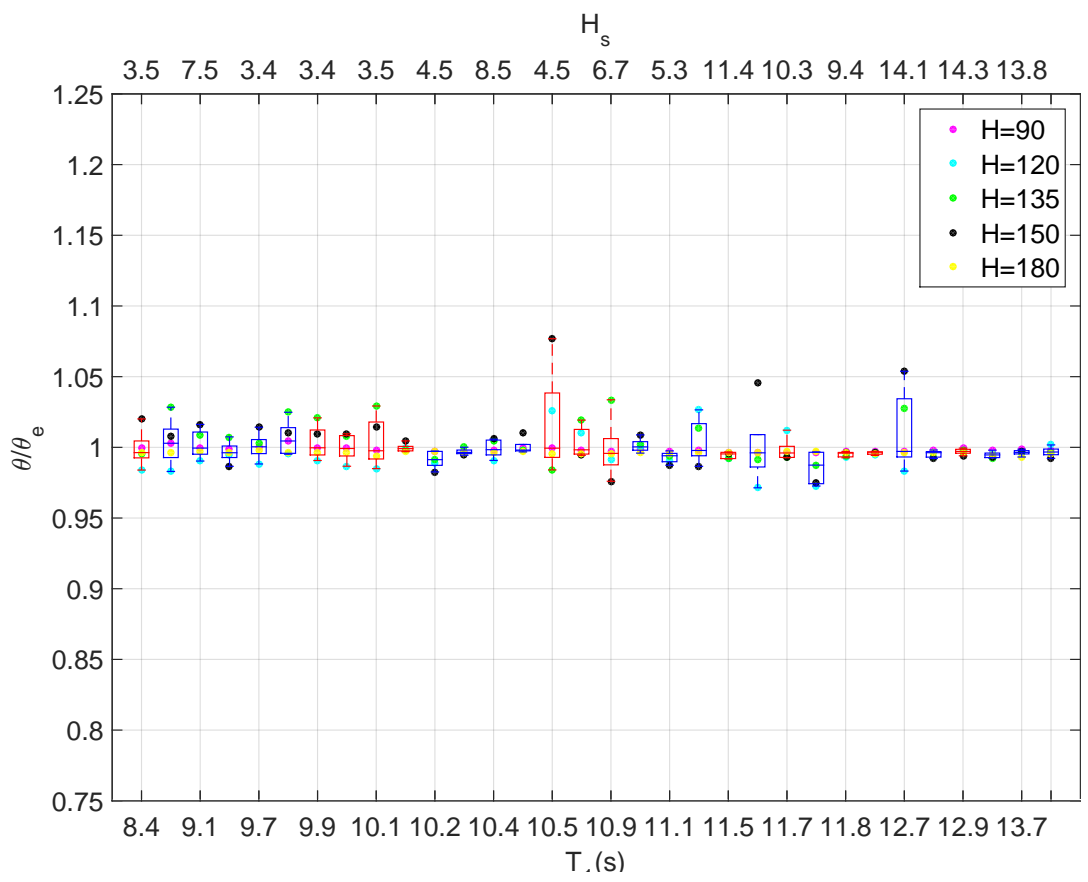

Figure 9. Box-plot showing the ratio between the estimated and measured mean wave direction, obtained by means of the alternative prior distribution.

For calm-to-mild sea conditions, the alternative prior distribution seems to result in a lower loss of energy than the conventional approach. As a matter of fact, Figure 8 shows a substantial improvement on the estimation of the significant wave height for sea conditions characterized with low mean wave periods, if compared with the results obtained with the conventional prior distribution and presented in Figure 5. 
A clear disadvantage that arises from the use of the alternative prior distribution is that it tends to overestimate the energy of the wave spectrum (or the significant wave height) in some cases. As a matter of fact, the alternative approach seems to be very sensitive to the heading for certain sea states, resulting in a significant increase of the variability, which results in differences up to $18 \%$ between the estimations of $H_{s}$ (worse than the ones obtained by means of the conventional approach). In a similar way, the estimations of the mean wave direction present the same pattern regarding the increment of variability of the estimation in accordance with the heading of the platform.

In theory, the alternative prior distribution provides a mechanism that avoids the estimation of spurious energy in the frequency and direction range close to the cancellation points. The reliability of this approach has been assessed by the improvement of the estimations of the directional energy spreading for sea conditions with mean periods above to $12.7 \mathrm{~s}$.

Figures 10 and 11 show a comparison between the estimations of the directional sea spectra, obtained by means of the two different approaches, the conventional approach and the Bayesian inference based on the alternative prior distribution. Regarding the directional spectra included in Figures 10 and 11, the wave energy in each wave frequency (radial coordinate in rad/s, with values given in real scale) is depicted for all wave directions (as a convention, the wave direction in the tank was considered as $180 \mathrm{deg}$ ). In addition, the main statistical parameters for each wave condition are presented at the top of each figure. The examples selected comprise one moderate sea condition, one mild sea condition and two extreme sea conditions (see Table 2).

The results obtained show that the energy spreading in the direction domain (the directional resolution considered in this study is equal to $\Delta \theta=5 \mathrm{deg}$ and the waves generated at the wave basin were long-crested) with the alternative prior distribution are in better agreement with the expected ${ }^{3}$ dispersion of the energy than the ones inferred using the conventional approach. However, the outputs of the alternative prior distribution are characterized by a non-smooth spectrum pattern for quartering sea conditions with small wave periods (for instance, see Figure 10). This can be linked to the fact that the alternative prior distribution no longer imposes the smoothness conditions as a necessary requirement of the solution, something that also leads to minor misleading estimations of the energy distribution in the direction. In any case, this drawback does not affect the energy distribution in the frequency-domain.

By inspecting these figures it can be concluded that the alternative approach, in general, provides solutions with a narrow spread of the energy in the direction. However, the use of the smoothness only as a weak condition, in the alternative prior distribution, may generate non-smooth solutions quartering seas. Another trend that can be identified is that spreading of the solutions obtained by means of both approaches are larger for extreme sea states than mild/calm sea states. This is due to the influence of the zero point that the heave RAO presents close to $22 \mathrm{~s}$. It is worth to remember that the alternative approach provides estimations characterized by a smaller spreading than the ones obtained through the conventional approach.

3 The measurements performed using an array of eight waves probes allow the estimation of the directional sea spectra by means of the Maximum Entropy Method (MEM), as described by [19]. 


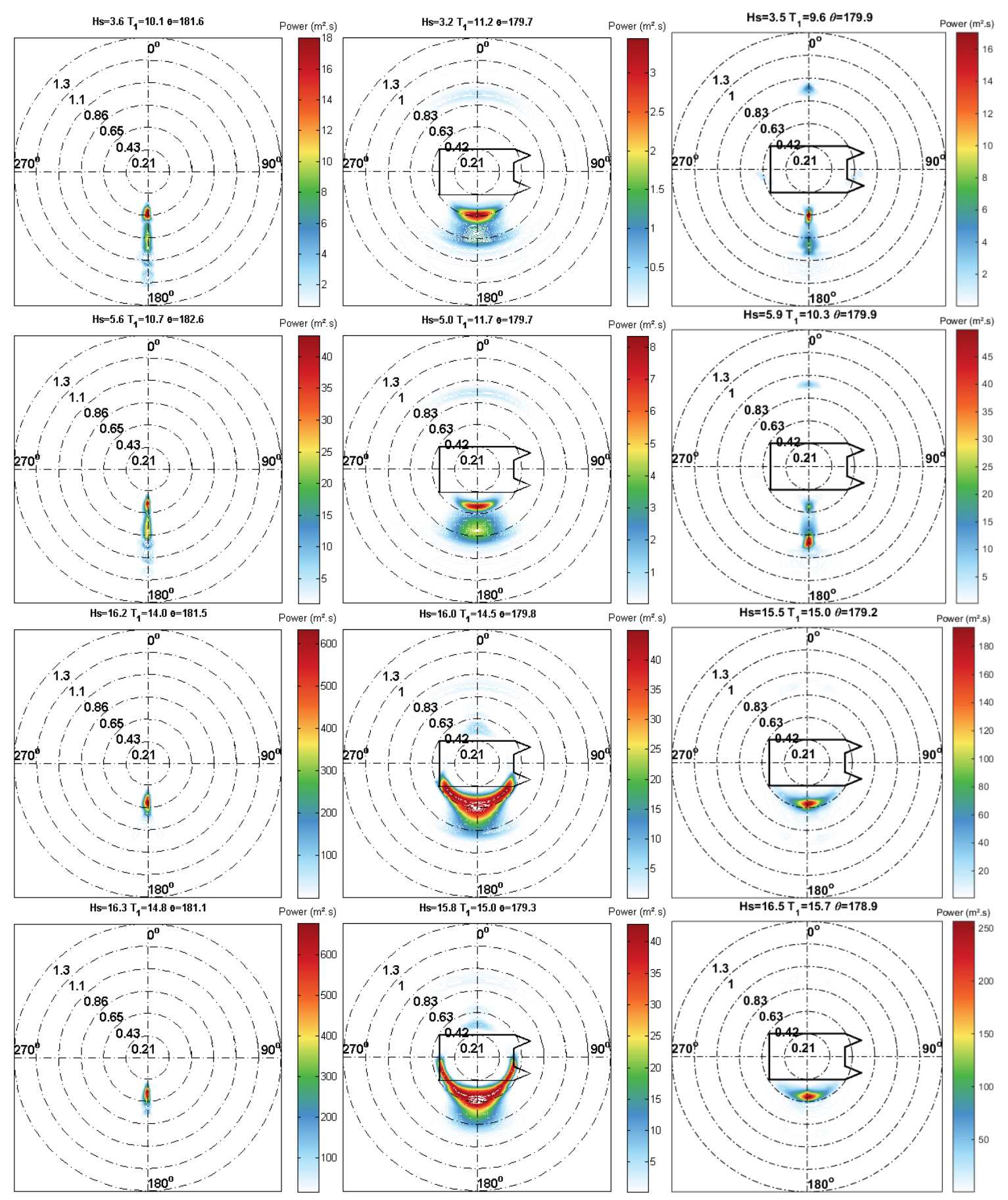

Figure 10. Comparison of directional wave spectra estimated with two different methods (for a heading equal to $90 \mathrm{deg}$.) and the paradigm. From left-to-right: Measured sea spectrum (MEM), the ones estimated with the conventional prior distribution and the output obtained with the alternative approach. From top-to-bottom: Sea conditions as provided Table 2. 


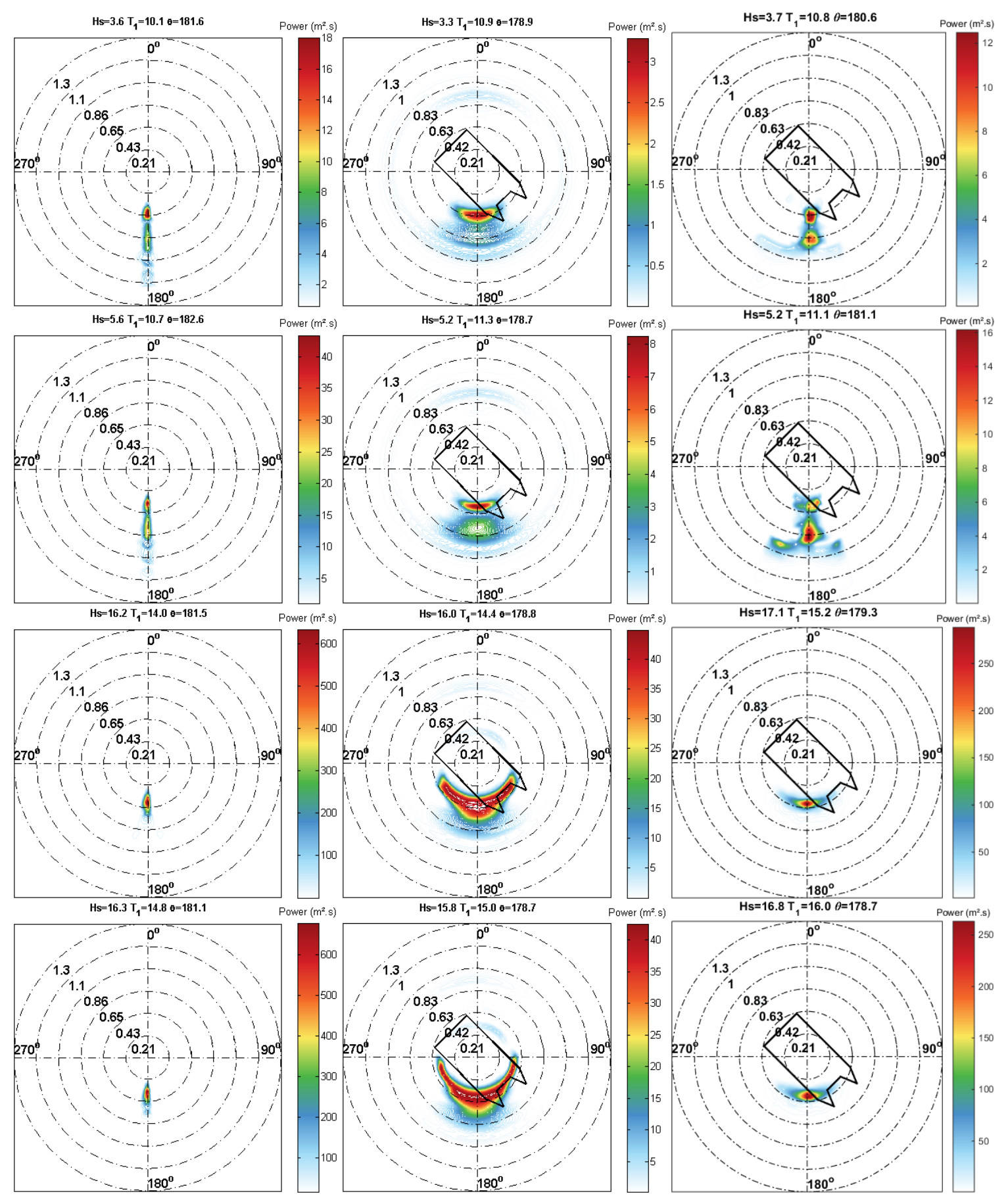

Figure 11. Directional wave spectra for a platform heading equal to 135 deg., estimated using three different methods. From left-to-right: Measured sea spectrum (MEM), the ones estimated with the conventional prior distribution and the output obtained with the alternative approach. From top-to-bottom: Sea conditions as provided Table 2. 
Table 2. Expected properties of the measured sea conditions (see [3]).

\begin{tabular}{ccccc}
\hline Test & Spectrum Type & $\boldsymbol{H}_{\boldsymbol{s}_{\boldsymbol{m}}}$ & $\boldsymbol{T}_{\boldsymbol{1}_{\boldsymbol{m}}}$ & Direction \\
\hline 6 & JONSWAP & 4.53 & 9.06 & $90 / 135^{\circ}$ \\
12 & Torsethaugen & 5.54 & 10.48 & $90 / 135^{\circ}$ \\
15 & JONSWAP & 7.50 & 9.60 & $90 / 135^{\circ}$ \\
30 & JONSWAP & 16.21 & 13.73 & $90 / 135^{\circ}$ \\
\hline
\end{tabular}

\section{Conclusions}

This work has primarily assessed the accuracy and the potential improvements of a proposed alternative prior distribution for Bayesian motion-based wave inference. The analysis performed was based on the results obtained through an extensive model-scale experimental campaign carried out at the USP's wave basin (TPN-USP), using a (1:120) scale model of an O\&G semisubmersible platform. In accordance with the objectives of this paper, the following conclusions have been drawn:

The comparison between outputs obtained with the conventional motion-based Bayesian wave inference approach and the ones resulting from the inference method proposed in this work has shown that the adoption of the later may lead to significant improvements. In fact, the error associated with the estimation of the mean wave period has been reduced in general by a factor equal to $5 \%$. Similarly, the estimation of the wave height revealed an improvement of the order of $3 \%$ regarding the mean error of the estimations, although it presents an increment of the error variability concerning different headings.

Once again, it is important to remember that the long-crested waves stand as a limiting condition for the conventional approach since it assumes a smooth distribution in the energy domain. Therefore, one may realize the relevance of the assessment regarding the performance of the methodologies adopted in this work by means of sea conditions generated (or measured) with a certain degree of spreading, something that has been left for future work.

To sum up, it can be concluded that the alternative prior distribution proposed brings some improvements if compared with the conventional approach. For instance, the alternative approach seems to provide more accurate estimations of sea conditions characterized by low mean wave periods. Similarly, the spreading of the energy in direction is narrower when the sea state is estimated by means of the alternative approach than if it is inferred using the conventional prior distribution. Nonetheless, the variance of the results, with regards to the different heading conditions, is increased when they are estimated though the alternative prior distribution.

Finally, another advantage obtained with the alternative inference method is the reduction of the computing time. As it has been pointed before, the computations were carried out with a desktop personal computer using MATLAB ${ }^{\circledR} 2014$ a. On average, the software took 28 s to estimate one sea condition using the conventional approach, without taking into account the time spent during the pre-calibration procedure of the hyperparameters. This meantime is reduced to only $6.7 \mathrm{~s}$ when the alternative prior distribution is adopted. This may be relevant for future applications of the method, such as its use to obtain a preliminary estimation of the sea state or even when large data sets are analyzed.

Author Contributions: Authors contributed equally to this work. All authors have read and agreed to the published version of the manuscript.

Funding: Jordi Mas-Soler thanks the scholarship received from the Coordenação de Aperfeiçoamento de Pessoal de Nível Superior-Brasil (CAPES)—Finance Code 001. Alexandre Nicolaos Simos acknowledges the Brazilian Research Council (CNPq) for his respective research grant. Antonio Souto-Iglesias and Jordi Mas-Soler also would like to thank the funding received from the ARCWIND project-Adaptation and implementation of floating wind energy conversion technology for the Atlantic region (EAPA 344/2016) —which is co-financed by the European Regional Development Fund through the Interreg Atlantic Area Programme

Acknowledgments: Authors are grateful to Petrobras and Equinor for supporting previous research on the VMB methodology, in the context of the regulations established by the National Petroleum Agency (ANP). USP's prior knowledge of the method contributed greatly to the motivation and execution of this work. 
Conflicts of Interest: The authors declare no conflict of interest. The funders had no role in the design of the study; in the collection, analyses, or interpretation of data; in the writing of the manuscript, or in the decision to publish the results.

\section{References}

1. Nielsen, U.D. Introducing two hyperparameters in bayesian estimation of wave spectra. Probabilistic Eng. Mech. 2008, 23, 84-94. [CrossRef]

2. Simos, A.N.; Tannuri, E.A.; da Cruz, J.J.; Queiroz Filho, A.N.; da Silva Bispo, I.B.; Carvalho, R.C. Development of an on-board wave estimation system based on the motions of a moored fpso: Commissioning and preliminary validation. In Proceedings of the 31st International Conference on Ocean, Offshore and Arctic Engineering, Rio de Janeiro, Brazil, 1-6 July 2012.

3. Mas-Soler, J.; Simos, A.N.; Tannuri, E.A. Estimating on-site wave spectra from the motions of a semisubmersible platform: An assessment based on model scale results. Ocean Eng. 2018, 153, $154-172$. [CrossRef]

4. Tannuri, E.A.; Sparano, J.V.; Simos, A.N.; Da Cruz, J.J. Estimating directional wave spectrum based on stationary ship motion measurements. Appl. Ocean Res. 2003, 25, 243-261. [CrossRef]

5. Nielsen, U.D.; Brodtkorb, A.H.; Sørensen, A.J. Sea state estimation using multiple ships simultaneously as sailing wave buoys. Appl. Ocean Res. 2019, 83, 65-76. [CrossRef]

6. de Souza, F.L.; Tannuri, E.A.; de Mello, P.C.; Franzini, G.; Mas-Soler, J.; Simos, A.N. Bayesian estimation of directional wave-spectrum using vessel motions and wave-probes: Proposal and preliminary experimental validation. J. Offshore Mech. Arct. Eng. 2018, 140, 2018. [CrossRef]

7. Bispo, I.B.; Queiroz Filho, A.N.; Tannuri, E.A.; Simos, A.N. Motion- based wave inference: Monitoring campaign on a turret fpso. In Proceedings of the 35th International Conference on Ocean, Offshore and Arctic Engineering, Busan, Korea, 19-24 June 2016.

8. Iseki, T.; Ohtsu, K. Bayesian estimation of directional wave spectra based on ship motions. Control Eng. Pract. 2000, 8, 215-219. [CrossRef]

9. Mas-Soler, J.; de Mello, P.C.; Tannuri, E.A.; Simos, A.N.; Souto- Iglesias, A. Impact of the unvertainties of the raos of a semisubmersible platform on the performance of a motion-based wave inference method. In Proceedings of the 38th International Conference on Ocean, Offshore and Arctic Engineering, Glasgow, UK, 9-14 June 2019.

10. Simos, A.N.; Tannuri, E.A.; Sparano, J.V.; Matos, V.L. Estimating wave spectra from the motions of moored vessels: Experimental validation. Appl. Ocean Res. 2010, 32, 191-208. [CrossRef]

11. Iseki, T. An improved stochastic modeling for bayesian wave estimation. In Proceedings of the 31st International Conference on Ocean, Offshore and Arctic Engineering, Rio de Janeiro, Brazil, 1-6 July 2012; pp. $455-461$.

12. Akaike, H. Likelihood and the bayes procedure. Trabajos de estadística y de Investigación Operativa 1980, 31, 143-166. [CrossRef]

13. Bui-Thanh, T. A Gentle Tutorial on Statistical Inversion Using the Bayesian Paradigm; Technical Report ICES-12-18; Institute for Computational Engineering and Sciences, The University of Texas at Austin: Austin, TX, USA, 2012.

14. Huber, P.J. A robust version of the probability ratio test. Ann. Math. Stat. 1965, 36, 1753-1758. [CrossRef]

15. Nielsen, U.D. Estimations of on-site directional wave spectra from measured ship responses. Mar. Struct. 2006, 19, 33-69. [CrossRef]

16. Mas-Soler, J. Assessing the Use of a Semisubmersible Oil Platform as a Motion-Based Sea Wave Sensor. Ph.D. Thesis, Escola Politécnica, University of São Paulo (USP), São Paulo, Brazil, 2018.

17. Torsethaugen, K.; Haver, S. Simplified double peak spectral model for ocean waves. In Proceedings of the 14th International Offshore and Polar Engineering Conference, Toulon, France, 23-28 May 2004. 
18. Lee, C.H.; Newman, J.N. Wamit User Manual; WAMIT, Inc.: Chestnut Hill, MA, USA, 2006.

19. Stansberg, C.T. On the fourier series decomposition of directional wave spectra. In Proceedings of the 8th International Offshore and Polar Engineering Conference, Montreal, CA, USA, 24-29 May 1998.

(C) 2020 by the authors. Licensee MDPI, Basel, Switzerland. This article is an open access article distributed under the terms and conditions of the Creative Commons Attribution (CC BY) license (http:/ / creativecommons.org/licenses/by/4.0/). 